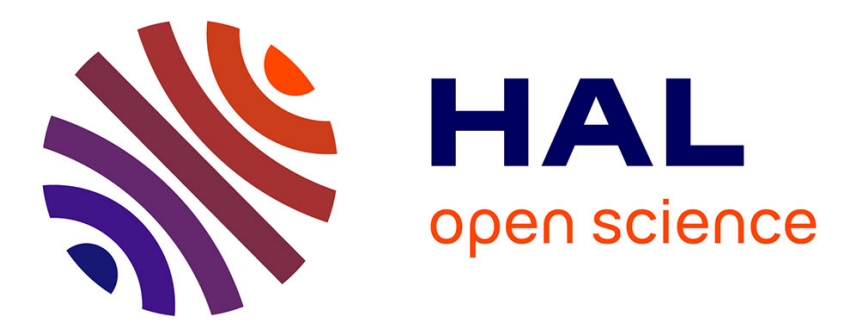

\title{
A tabulated chemistry method for spray combustion
}

\author{
B. Franzelli, B. Fiorina, N. Darabiha
}

\section{To cite this version:}

B. Franzelli, B. Fiorina, N. Darabiha. A tabulated chemistry method for spray combustion. Proceedings of the Combustion Institute, 2013, 34 (1), pp.1659-1666. 10.1016/j.proci.2012.06.013 . hal01272971

\section{HAL Id: hal-01272971 \\ https://hal.science/hal-01272971}

Submitted on 13 Mar 2017

HAL is a multi-disciplinary open access archive for the deposit and dissemination of scientific research documents, whether they are published or not. The documents may come from teaching and research institutions in France or abroad, or from public or private research centers.
L'archive ouverte pluridisciplinaire HAL, est destinée au dépôt et à la diffusion de documents scientifiques de niveau recherche, publiés ou non, émanant des établissements d'enseignement et de recherche français ou étrangers, des laboratoires publics ou privés. 


\title{
A tabulated chemistry method for spray combustion
}

\author{
B. Franzelli ${ }^{\mathrm{a}, \mathrm{b}, *}$, B. Fiorina ${ }^{\mathrm{a}, \mathrm{b}}$, N. Darabiha ${ }^{\mathrm{a}, \mathrm{b}}$ \\ ${ }^{a}$ Ecole Centrale Paris, Grande Voie des Vignes, 92290 Châtenay-Malabry, France \\ ${ }^{b}$ CNRS, UPR 288, Laboratoire d'Energétique Moléculaire et Macroscopique, Combustion \\ (EM2C), Grande Voie des Vignes, 92290 Châtenay-Malabry, France
}

\begin{abstract}
Tabulated chemistry is a popular technique to account for detailed chemical effects with an affordable computational cost in gaseous combustion systems. However its performances for spray combustion have not completely been identified. The present article discusses the chemical structure modeling of spray flames using tabulated chemistry methods under the hypothesis that the chemical subspace accessed by a two-phase reactive flow can be mapped by a collection of gaseous flamelets. It is shown that tabulated chemistry methods based either on pure premixed flamelets or on pure non-premixed flamelets fail to capture the structure of spray combustion. The reason is the complexity of the chemical structure of spray flames which exhibits both premixed-like and non-premixed-like reaction zones. To overcome this issue, a new multi-regime flamelet combustion model (called Partially-Premixed Flamelet Tabulation 2PFT) is presented in this paper. Information from premixed, partially-premixed and diffusion flames are stored in a 3-D look-up table parametrized as function of the progress variable $Y_{c}$, describing the progress of the reaction, the mixture fraction $Y_{z}$, denoting the lo-
\end{abstract}

\footnotetext{
${ }^{*}$ Corresponding author

Email address: benedetta.franzellidecp.fr (B. Franzelli)
} 
cal equivalence ratio, and the scalar dissipation $\chi^{*}$, which identifies the combustion regime. The performances of the 2PFT method are evaluated on counterflow laminar spray flames for different injection conditions of droplet diameter, liquid volume fraction and velocity. The 2PFT tabulation method better describes the chemical structure of spray flames compared to the classical techniques based on single archetypal flamelets. These results also confirm that the chemical structure of laminar spray flame can be modeled by a multi-regime flamelet combustion model based on gaseous flamelets.

Keywords: tabulated chemistry, spray combustion, combustion modeling, partially-premixed combustion. 


\section{Introduction}

A complete comprehension of spray combustion is necessary to guarantee flame stabilization and to reduce pollutant emissions in aircraft combustions and internal combustion engines [1]. Spray combustion comprises liquid dispersion into droplets and evaporation and presents a complex chemical structure since the fuel droplet evaporation process causes strong inhomogeneities of equivalence ratio in the fresh gases $[2,3]$. For these reasons, the numerical prediction of combustion phenomena sensitive to detailed chemistry, such as the pollutant predictions or the flame propagation, is very challenging.

Unfortunately, the use of detailed kinetic mechanisms is prohibitive in turbulent combustion simulation of complex industrial configurations due to their too high computational cost and stiffness. Commonly-used approaches to overcome this issue are flamelet-based tabulated chemistry methods that have initially been developed to introduce detailed chemistry in CFD simulation of gaseous flames at a reduced computational cost. The turbulent flame structure is modeled by an ensemble of single flamelet elements generally assuming a single burning regime. However, this assumption may be too restrictive. Premixed Flamelet Tabulation (PFT) methods $[4,5]$, for example, are based on premixed flamelets and are strictly valid only in premixed combustion regimes while Diffusion Flamelet Tabulation (DFT) techniques rely on diffusion flamelets and are adapted for non-premixed combus-

tion [6, 7]. Only recently, multi-regime flamelet methods $[8,9,10,11]$ have been proposed to model the structure of complex gaseous flames where both premixedlike and diffusion-like reactive layers are present such as in multi-injection gas turbines or spray combustors.

Although being attractive, the performances of tabulated chemistry method have 
never been completely investigated for spray combustion. Hollmann and Gutheil [12] observed that a laminar gas library based on a single combustion regime cannot correctly reproduce the structure of a spray flame since premixed-like, partiallypremixed-like or diffusion-like features could be found depending on the boundary conditions. To capture these complex regimes, they proposed a tabulated chemistry method directly based on spray flamelets. This strategy is consistent but requires a very high-dimensional library as all physical spray parameters have to be varied: droplet diameter, liquid volume fraction, liquid injection velocity,... A more efficient solution would be to tabulate only the chemical subspace effectively accessed by spray combustion. A possible strategy is therefore to identify this subspace from gaseous flame elements as in $[4,5,10,13]$. This point has already been evidenced by Baba and Kurose [14] who mentioned the need of a combustion model for partially-premixed flames to guarantee the prediction accuracy of spray jet flames.

The present article discusses the chemical structure modeling of spray flames using tabulated chemistry methods, relying on the assumption that the chemical subspace accessed by a two-phase reactive flow can be mapped by a collection of gaseous flamelets. Different tabulated chemistry strategies will be tested. In Section 2, the investigated configuration, a 1-D axisymmetric conterflow spray flame, is described. Three tabulated chemistry strategies are then introduced in Section 3. The PFT methods, based on premixed flamelets, the DFT approach, based on diffusion flamelet elements, and a new technique called PartiallyPremixed Flamelet Tabulation (2PFT). The 2PFT method, based on the tabulation of partially-premixed flamelets, is proposed here to tackle complex chemical spray flame structure. All tabulation techniques are tested in Section 4.1 on the 
1-D counterflow spray flame configuration. As the structure of spray flames is highly sensitive to the boundary conditions, the performances of tabulated chemistry methods for spray combustion are finally assessed in Section 4.2 for different values of injection velocity, droplet diameter and liquid volume fraction.

\section{Numerical description of a spray flame}

In the following, an axisymmetric mono-disperse counterflow spray flame is considered [15]. Pure fresh air is injected on the left side (identified by superscript $o x$ ) whereas spray fuel and gaseous air are injected on the right side of the configuration (superscript $f$ ). The gas and liquid phases at the right inlet have the same temperature $T_{g}^{f}=T_{l}^{f}$ and axial velocity $v_{g}^{f}=v_{l}^{f}$, where subscripts $g$ and $l$ denote the gaseous and the liquid phase respectively. The same values of temperature and axial velocity are imposed at both injection sides: $T_{g}^{o x}=T_{g}^{f}$ and $v_{g}^{o x}=v_{g}^{f}$.

Accordingly to [16], it could be shown that on the jet axis the mass fraction of the $k^{\text {th }}$ species $Y_{k}$ as well as the density $\rho_{g}, \rho_{l}$, the axial velocity $v_{g}, v_{l}$ and the temperature $T_{g}, T_{l}$ only depend on the axial coordinate $x$ for both gas and liquid phases. The radial velocities depend also on the radial coordinate $r: u_{g}=r U_{g}(x)$ and $u_{l}=r U_{l}(x)$.

Assuming a constant pressure gradient in the radial direction $\frac{1}{r} \frac{\partial p}{\partial r}=-J$, the gas phase flow is described by the balance equations for mass, radial and axial 
momentum, energy and species [15]:

$$
\begin{aligned}
2 \rho_{g} U_{g}+\frac{\partial \rho_{g} v_{g}}{\partial x}= & n_{l} \dot{m}_{l} \\
\rho_{g} U_{g}^{2}+\rho_{g} v_{g} \frac{\partial U_{g}}{\partial x}= & \frac{\partial}{\partial x}\left(\mu_{g} \frac{\partial U_{g}}{\partial x}\right)+J \\
& +n_{l} \dot{m}_{l}\left(U_{l}-U_{g}\right)-n_{l} \frac{f_{r}}{r} \\
\rho_{g} v_{g} c_{p_{g}} \frac{\partial T_{g}}{\partial x}= & \frac{\partial}{\partial x}\left(\lambda_{g} \frac{\partial T_{g}}{\partial x}\right)-\sum_{k=1}^{K} h_{k} W_{k} \dot{\Omega}_{k} \\
& -\left(\sum_{k=1}^{K} \rho_{g} Y_{k} V_{k x} c_{p_{g k}}\right) \frac{\partial T_{g}}{\partial x} \\
& +n_{l} \dot{m}_{l} c_{p_{g F}}\left(T_{l}-T_{g}\right)-n_{l} \dot{m}_{l} q \\
\rho_{g} v_{g} \frac{\partial Y_{k}}{\partial x}= & -\frac{\partial}{\partial x}\left(\rho_{g} Y_{k} V_{k x}\right)+W_{k} \dot{\Omega}_{k} \\
& +\left(\delta_{k F}-Y_{k}\right) n_{l} \dot{m}_{l},
\end{aligned}
$$

where $n_{l}$ is the droplet number density, $\dot{m}_{l}$ is the mass vaporization rate of a single droplet, $\mu_{g}$ is the mixture viscosity,$f_{i}$ is the $i^{\text {th }}$ component of the drag force modeled by the Stokes law, $c_{p_{g k}}$ and $c_{p_{g}}$ are the heat capacity at local constant pressure of species $k$ and of the mixture respectively, $h_{k}, W_{k}, \dot{\Omega}_{k}$ are the specific enthalpy, the molar weight and the molar chemical production rate of the $k^{\text {th }}$ species respectively, $V_{k x}$ is the diffusion velocity of the $k^{\text {th }}$ species in the axial direction, $q$ is the heat transfered from the gas to each droplet and $\delta_{k F}$ is the Dirac's delta equal to one only for $k=F$ (where subscript $\mathrm{F}$ is the index for fuel species). 
The liquid phase flow is obtained solving the following conservation equations [15]:

$$
\begin{aligned}
v_{l} \frac{\partial m_{l}}{\partial x} & =-\dot{m}_{l}, \\
m_{l} U_{l}^{2}+m_{l} v_{l} \frac{\partial U_{l}}{\partial x} & =\frac{f_{r}}{r}, \\
m_{l} v_{l} \frac{\partial v_{l}}{\partial x} & =f_{x}, \\
m_{l} c_{p_{l}} v_{l} \frac{\partial T_{l}}{\partial x} & =\dot{m}_{l}(q-L), \\
2 n_{l} U_{l}+\frac{\partial n_{l} v_{l}}{\partial x} & =0,
\end{aligned}
$$

where $m_{l}=\frac{4}{3} \pi R_{l}^{3} \rho_{q}$ is the mass of a single droplet (with $R_{l}$ the droplet radius and $\rho_{q}$ the liquid specific mass), $L$ is the latent heat of evaporation and $c_{p_{l}}$ is the constant pressure heat capacity of the liquid species. The system of equations is completed by assuming a constant radial pressure-gradient in the axial direction $\frac{d J}{d x}=0$. The mass vaporization rate of a single droplet $\dot{m}_{l}$ and the heat transferred from the gas to each droplet $q$ are modeled by the expression for a spherically symmetric single-component droplet obtained from the film temperature model [17].

\section{Tabulation methods for counterflow spray flames}

Tabulated chemistry is a popular technique to account for detailed chemical effects with a reasonable computational cost [18]. Among the different strategies to generate chemical look-up tables, some of them are based on physical considerations like the PFT methods $[4,5]$, based on premixed flamelets, and DFT techniques $[6,7]$ relying on diffusion flamelets. These techniques assume that the chemical flame structure is described in a reduced phase subspace from elementary configuration. For instance, PFT and DFT models assume that the chemical 
trajectories accessed in an adiabatic stratified reactive environment evolve in a 2 D subspace $\left(Y_{c}, Y_{z}\right)$, where $Y_{c}$ and $Y_{z}$ are the progress variable and the mixture fraction respectively. The progress variable $Y_{c}$ evolves monotonically between fresh and burnt gases. An explicit definition is [19]:

$$
Y_{C}=Y_{C O}+Y_{C O_{2}}
$$

The mixture fraction $Y_{z}$ is defined from a linear combination of carbon elements in order to represent the local fuel/air mixing:

$$
Y_{z}=\sum_{k=1}^{N_{\text {spec }}} n_{C k} \frac{Y_{k} W_{C}}{W_{k}},
$$

where $W_{C}$ is the carbon element weight and $n_{C k}$ is the number of carbon atoms of the $k^{\text {th }}$ species. This assumption is valid when the flame structure remains close to the single flamelet archetype used to construct the chemical structure. However, as shown in $[10,13]$, there exists more complex situation where effective dimension of the accessed composition space is higher. Supplementary coordinates such as the scalar dissipation rate for the mixture fraction and/or for the progress variable should be added to capture partially-premixed flames where both premixed-like and non premixed-like reaction zones exist.

In the following, we will first describe flamelet tabulation technique that assumes chemical system evolution in a 2-D subspace. Two formulations are represented where chemistry is tabulated from premixed unstrained flamelets and counterflow non-premixed flamelets, respectively. Next, we present an enhanced tabulated chemistry method called 2PFT that increases the range of validity of the model by adding a coordinate to the chemical look-up table, the mixture fraction scalar 
dissipation rate, as suggested by [10].

\subsection{Premixed Flamelet Tabulation (PFT)}

PFT methods, such as FPI [4] or FGM [5], assume that the chemical subspace accessed by a flame can be mapped by a collection of 1-D gaseous laminar premixed flames computed using detailed chemistry for various equivalence ratios $\phi$ within the range $\left[\phi_{L}, \phi_{R}\right]$, where $\phi_{L}$ and $\phi_{R}$ are the lean and rich flammability limits. Any thermo-chemical quantity $\varphi$ is stored in a 2-D look-up table: $\varphi=\varphi^{P F T}\left[Y_{c}, Y_{z}\right]$, where $\varphi^{P F T}$ is computed from the collection of 1-D premixed flamelets.

The rich $\phi_{\mathbf{R}}$ (lean $\phi_{\mathbf{L}}$ ) flammability limit is defined as the maximal (minimal) possible value of the equivalence ratio of a premixed flame. This approach is accurate when the modeled flame structure remains close to the structure of a premixed flame front but introduces errors when diffusion of mass through iso-equivalence ratio dominates [20].

\subsection{Diffusion Flamelet Tabulation (DFT)}

A possible alternative is to tabulate the chemistry from diffusion flamelets as in the flamelet model [6] or in FPV method [7]. In the DFT method, any thermo-chemical quantity $\varphi$ is obtained from a look-up table built from gaseous strained diffusion flames for different values of the strain rate $0<a<a^{e x t}$, where $a^{e x t}$ is the extinction limit, by varying the inlet velocities. ${ }^{1}$ As in PFT method, thermo-chemical variables can be mapped in the 2-D subspace $\left(Y_{c}, Y_{z}\right)$ :

\footnotetext{
${ }^{1}$ In a first approximation, the strain rate $a$ of a counterflow flame is estimated from the inlet velocities and jet distance $H: a=\frac{v^{o x}+v^{f}}{H}$.
} 
$\varphi=\varphi^{D F T}\left[Y_{c}, Y_{z}\right]$. This approach is dedicated to diffusion flames but is not able to capture the structure and consequently the propagation of premixed flame front.

\subsection{Partially-Premixed Flamelet Tabulation (2PFT)}

In the 2PFT method, it is assumed that the chemical flame structure of a complex flame is mapped by a collection of 1-D gaseous partially-premixed counterflow flamelets characterized by an injection of pure air against a fuel/air mixture for different values of equivalence ratio $\phi^{f}$ and strain rate $a$.

The table is then built by varying the fuel/air stream equivalence ratio between $\phi^{f}=\phi_{L}^{f}$ and $\phi^{f}=+\infty$, where $\phi_{L}^{f}$ is the smallest value of the fuel/air stream equivalence ratio for which the counterflow flame is stabilized, and solving the flamelet for different values of the strain rate $0<a<a^{e x t}$. The limits of the 2PFT table tend towards PFT and DFT tables respectively. Indeed when $\phi^{f}=+\infty$ and $0<a<a^{e x t}$ the table is filled by diffusion flamelets as in DFT. At the opposite when $a=0$ and $\phi_{L}<\phi^{f}<\phi_{R}$ the table is filled with unstrained laminar premixed flames as in PFT. The 2PFT method is then based on different archetypal flamelets to map the subspace accessed by premixed, non-premixed and partiallypremixed flames. This subspace is identified by three parameters: the progress variable $Y_{c}$, the mixture fraction $Y_{z}$ and the scalar dissipation rate $\chi^{*}$ of $Y_{z}$ :

$$
\chi^{*}=\rho_{0} D_{0}\left|\nabla Y_{z}\right|^{2}
$$

where $\rho_{0}$ and $D_{0}$ are a reference density and diffusion coefficient, respectively. In the present work, they correspond to those of a stoechiometric kerosene/air mixture at atmospheric pressure and temperature $T_{0}=400 \mathrm{~K}$. Each flamelet point has a unique representation in the $\left(Y_{c}, Y_{z}, \chi^{*}\right)$ subspace. Any thermo-chemical 
quantity $\varphi$ can then be stored in a 3-D look-up table: $\varphi=\varphi^{2 P F T}\left[Y_{c}, Y_{z}, \chi^{*}\right]$.

Other strategies are possible to generate the chemical table adapted to partiallypremixed combustion. Among them, are the REDIM[13] and the multi-dimensional flamelet-generated manifold [10] models. These approaches directly solve a projection of the full system of mass conservation species and energy balance equations in the $\left(Y_{c}, Y_{z}\right)$ subspace. Models are required for the progress variable and the mixture fraction dissipation rates. The 2PFT method proposed here is quite different as the table is built from counterflow partially-premixed flamelet solutions obtained in the physical space. Moreover, compared to other multi-regime flamelet models $[8,19,21]$ the 2 PFT method does not require a flame index, i.e. a model to distinguish between combustion regimes, since the scalar dissipation rate $\chi^{*}$ identifies the combustion regime, i.e. from $\chi^{*}=0$ for premixed flames through the maximum values correspond to diffusion flames.

\section{Results on spray flames}

The performances of PFT, DFT and 2PFT strategies are now investigated in spray flames. The structure of a counterflow spray flame strongly depends on the gaseous flow properties as well as the characteristics of the spray, such as the droplet diameter $D_{l}$, the liquid temperature $T_{l}$ and the liquid volume fraction $\alpha_{l}=\frac{4}{3} \pi n_{l}\left(\frac{D_{l}}{2}\right)^{3}$. The capability of the previously introduced methods to capture the structure of a kerosene spray flame is investigated here for the cases summarized in Table 1. All spray flames have been simulated with the REGATHcounterflow code [22] using a detailed mechanism for kerosene/air flame comprising $N_{\text {spec }}=74$ species and $N_{\text {reac }}=991$ reactions [23]. To simplify the calculation, kerosene is represented by the $\mathrm{C}_{10} H_{22}$ species, whose liquid charac- 
teristics are summarized in Table 2, and unity Lewis number is assumed for all species.

A first reference spray flame is computed with the following injection conditions: $D_{l}^{f}=40 \mu \mathrm{m}, \alpha_{l}^{f}=3.010^{-3}, v_{g}^{f}=v_{l}^{f}=0.20 \mathrm{~m} / \mathrm{s}$ and $T_{g}^{f}=T_{l}^{f}=400 \mathrm{~K}$. The jet distance is $H=0.02 \mathrm{~m}$. Figure 1 plots the temperature and the evaporation source term along the spatial coordinate $x$. The evaporation source term $n_{l} \dot{m}_{l}$ is high near the right injection of liquid kerosene. It presents a peak near the flame front $(x \approx 0.4 \mathrm{~cm})$, where the gas temperature increases due to the thermal conductivity. Species mass fractions are plotted in Fig. 2 and show that this high temperature region $(-0.4 \mathrm{~cm}<x<0.4 \mathrm{~cm})$ is characterized by the presence of intermediate species and products. The burnt products are then diluted by the opposed air jet. The production rate of the progress variable $\dot{\omega}_{Y_{c}}=W_{C O} \dot{\Omega}_{C O}+W_{C_{2}} \dot{\Omega}_{C O_{2}}$ (in $\mathrm{kg} / \mathrm{m}^{3} / \mathrm{s}$ ) is extremely important since it incorporates all the chemical information in the tabulation methods. The chemical source term $\dot{\omega}_{Y_{c}}$ of the reference spray counterflow flame obtained with the detailed mechanism is plotted in Fig. 3a (continuous line). It presents two peaks. A first reaction region is found for $0<x<0.2 \mathrm{~cm}$, where fresh kerosene and air burn in a premixed-like regime. A second reaction zone is observed at $x \approx-0.05 \mathrm{~cm}$ where burnt products like $\mathrm{CO}$ recombine into $\mathrm{CO}_{2}$ with fresh air coming from the left side (cfr. Fig. 2). As a consequence, to correctly capture the whole structure of a kerosene spray flame the tabulation method is expected to describe both diffusion-like and premixedlike features.

\subsection{A priori testing}

The performances of the three previously described tabulation methods are evaluated on the reference kerosene spray flame with an a priori test. 
The PFT, DFT and 2PFT look-up tables are built from gaseous flamelet solutions obtained with the REGATH package and keeping the same detailed mechanism used for the spray flame calculations. The PFT table is built from a collection of unstrained adiabatic gaseous premixed flamelets for $\phi_{L}<\phi<\phi_{R}$ while the DFT table is based on adiabatic gaseous counterflow diffusion flamelets computed for different values of the strain rate $0<a \leq a_{\text {ext }}$ by varying the injection velocity between $0.2 \mathrm{~m} / \mathrm{s}$ and $10.0 \mathrm{~m} / \mathrm{s}$. The $2 \mathrm{PFT}$ table is composed by a collection of adiabatic gaseous counterflow flamelets for 10 different values of fuel stream equivalence ratio: $1.6<\phi^{f} \leq+\infty\left(0.1<Y_{C 10 H 22}^{f} \leq 1.00\right)$ and for different values of the strain rate by varying the injection velocity within the extinction limit. In all flamelets, the fresh gas inlet temperature is equal to the injection temperature of spray flames $(T=400 K)$. In the investigated cases, heat exchanges due to kerosene evaporation between liquid and gas phases impact very slightly the solution. Adiabaticity is therefore assumed to simplify the tabulation procedure. The performances of the tabulated mechanisms are evaluated through an a priori analysis that compares the tabulated quantities to the reference solution obtained with the detailed scheme. For each point of the detailed mechanism solution, the values of progress variable $\tilde{Y}_{c}$, mixture fraction $\tilde{Y}_{z}$ and $\tilde{\chi}^{*}$ are evaluated using Eqs. (10)-(11) and (12). The tabulated chemical source terms are then extracted from the PFT, DFT and 2PFT look-up tables $\left(\dot{\omega}_{Y_{c}}{ }^{P F T}\left[\tilde{Y}_{c}, \tilde{Y}_{z}\right],{\dot{\omega_{Y_{c}}}}^{D F T}\left[\tilde{Y}_{c}, \tilde{Y}_{z}\right]\right.$ and $\dot{\omega}_{Y_{c}}{ }^{2 P F T}\left[\tilde{Y}_{c}, \tilde{Y}_{z}, \tilde{\chi}^{*}\right]$ respectively) and compared to the reference $\dot{\omega}_{Y_{c}}$ obtained with the detailed chemistry in Fig. 3a (PFT-dotted line, DFT-crosses, 2PFT-circles). The PFT method correctly predicts $\dot{\omega}_{Y_{c}}$ in the first premixed-like reaction zone, whereas it is not able to reproduce the recombination zone between $\mathrm{CO}$ and $\mathrm{CO}_{2}$. The DFT method globally overestimates $\dot{\omega}_{Y_{c}}$ The 2PFT methodology is able to 
accurately describe the production rate in the whole reaction region characterized by both diffusion and premixed combustion regimes. The $\mathrm{CO}$ mass fraction is also extracted from the look-up tables and is compared to the detailed chemistry solution in Fig. 3b.. As already observed for the production rate $\dot{\omega}_{Y_{c}}$, the 2PFT method better reproduce the flame structure over the whole computational domain compared to the classical techniques.

\subsection{Parametric analysis}

The ability of the 2PFT tabulation method to correctly describe the flame structure of a spray flame is also evaluated for the different fuel spray injection conditions of droplet diameter $D_{l}^{f}$, liquid volume fraction $\alpha_{l}^{f}$ and velocity $v_{g}^{f}=v_{l}^{f}$. All investigated cases are summarized in Table 1.

In the cases DIAMa and DIAMb, the droplet diameter is equal to $D_{l}^{f}=70 \mu \mathrm{m}$ and $D_{l}^{f}=140 \mu \mathrm{m}$ respectively while the injected liquid fuel quantity, i.e. the liquid volume fraction $\alpha_{l}$, is kept constant by modifying the droplet density number $n_{l}$. The progress variable reaction rates are compared to the reference solution $\dot{\omega}_{Y_{c}}$ for $D_{l}^{f}=70 \mu m$ (Fig. 4). It is composed by a premixed region and a recombination zone as in the reference case. For a higher droplet diameter $\left(D_{l}^{f}=140 \mu \mathrm{m}\right)$, evaporation is initially slower and the kerosene remains mainly in liquid phase before entering into the flame front where it evaporates rapidly. The flame mainly presents one rich partially-premixing-like reaction zone that the PFT method is not able to reproduce. On the contrary, the DFT and 2PFT methodologies fairly agree with the reference solution.

In ALPHAa and ALPHAb cases, the liquid volume fraction $\alpha_{l}^{f}$ is decreased keeping constant the droplet diameter $\left(D_{l}^{f}=40 \mu \mathrm{m}\right)$. The reconstructed progress 
variable reaction rates are compared in Fig.5 to the detailed mechanism solutions. For $\alpha_{l}^{f}=1.410^{-3}$ the second reaction zone observed at $-0.1 \mathrm{~cm}<x<0.1 \mathrm{~cm}$ is characterized by a less intense reaction rate $\dot{\omega}_{Y_{c}}$ whereas the intensity of $\dot{\omega}_{Y_{c}}$ of the first premixed-like region is higher. For a lower value $\alpha_{l}^{f}=0.6810^{-3}$, the evaporation zone is thinner and located closer to the injection $(x>0.6 \mathrm{~cm})$. No second diffusion or partially-premixed reaction zone is found. Once again, the 2PFT method is able to correctly capture the different flame structures.

For VELa and VELb cases, the injection velocity is increased $\left(D_{l}^{f}=40 \mu \mathrm{m}, \alpha_{l}^{f}=\right.$ $\left.3.010^{-3}\right)$ and, as a consequence, the flame strain rate increases too $\left(v_{g}^{f}=v_{l}^{f}=\right.$ $0.5 \mathrm{~m} / \mathrm{s}$ and $v_{g}^{f}=v_{l}^{f}=1.0 \mathrm{~m} / \mathrm{s}$ ). For these flames, the evaporation zone overlaps a smaller reaction zone. Both the PFT and the DFT methods mainly capture the production rate ${\dot{\omega_{Y}}}$ for the highest value of $v_{g}^{f}$ but the 2PFT method better predicts the flame structure even for smaller strain rate values.

In all studied cases, it has been shown that the 2PFT tabulation method based on gaseous partially-premixed flamelets is able to correctly describe the structure of the spray flames which are characterized by different combustion regimes.

\section{Conclusion}

An evaluation of tabulation technique for spray combustion has been presented in this article. The new chemical 2PFT method has been proposed to enhance the range of validity of tabulation methods to multi-regime combustion. It is based on gaseous premixed, partially-premixed and diffusion flamelets parametrized by the progress variable $Y_{c}$, describing the progress of the reactions, the mixture fraction $Y_{z}$, denoting the local equivalence ratio, and the scalar dissipation rate $\chi^{*}$, identifying the combustion regime. Results have been compared to those of the classical 
tabulated chemistry PFT and DFT methods on spray flames using a priori tests for different values of droplet diameter, liquid volume fraction and injection velocity. In all tested cases, the 2PFT tabulation method better describes the flame structure compared to the classical techniques based on single archetypal flamelets and it is more adequate for counterflow spray flames. It has also been demonstrated that the chemical structure of laminar spray flames could be modeled by a tabulated multi-regime flamelet combustion regime based on gaseous flamelets.

Nevertheless, the 2PFT method has to be validated on more test cases varying the injection temperature as well as for higher droplet diameters that could eventually pass through the flame front. Moreover, variations of fresh mixture enthalpy are expected to have an important role on spray flames for some inlet conditions and their integration in the 2PFT table has to be deeply investigated.

\section{Acknowledgments}

The research leading to these results has received funding from the European Communitys Seventh Framework Programme (FP7/2007-2013) under Grant Agreement \# ACP8-GA-2009-234009 (KIAI project). The KIAI project started in May 2009 for a duration of 4 years and addresses innovative solutions for the development of new combustors in aero-engines. It aims at providing low NOx methodologies to be applied to design these combustors.

\section{References}

[1] X. Jiang, G. A. Siamas, K. Jagus and T. G. Karaylannis. Prog. Energy Comb. Sci., 36(2): $131-167,2010$.

[2] E. Gutheil and W. A. Sirignano. Combust. Flame, 113:92-105, 1998.

[3] H. Watanabe, R. Kurose, S. M. Hwang and F. Akamatsu. Combust. Flame, 148:234-248, 2007. 
[4] O. Gicquel, N. Darabiha and D. Thévenin. Proceedings of the Combustion Institute, 28: 1901-1908, 2000.

[5] J. A. van Oijen, F. A. Lammers and L. P.H. de Goey. Combust. Flame, 127:2124-2134, 2001.

[6] N. Peters. Prog. Energy Comb. Sci., 10:(3) 319-339, 1984.

[7] C. Pierce and P. Moin. J. Fluid Mech., 504:73-97, 2004.

[8] E. Knudsen and H. Pitsch. Combust. Flame, 159:242-264, 2012.

[9] J. A. van Oijen and L. P.H. de Goey. Combust. Theory and Modelling, 8:141-163, 2004.

[10] P. D. Nguyen, L. Vervisch, V. Subramanian and P. Domingo. Combust. Flame, 157:43-61, 2010.

[11] W. J.S. Ramaekers, J. A. van Oijen and L. P.H. de Goey. Flow, Turb. and Combustion, 84 (3):43-61, 2010.

[12] C. Hollmann and E. Gutheil. Combust. Sci. Tech., 135:175-192, 1998.

[13] V. Bykov and U. Maas. Combust. Theory and Modeling, 11(6):839-862, 2007.

[14] Y. Baba and R. Kurose. J. Fluid Mech., 612:45-79, 2008.

[15] N. Darabiha, F. Lacas, J. C. Rolon and S. Candel. Combust. Flame, 95(3):261-275, 1993.

[16] G. Contillo and W. A. Sirignano Combust. Flame, 81:325-340, 1990.

[17] K. K. Kuo. Principles of Combustion, John Willey and Sons, 2005.

[18] U. Mass and S. B. Pope. Twenty-Fourth Symposium (International) on Combustion, 24(1): 103-112, 1992.

[19] B. Fiorina, O. Gicquel, L. Vervisch, S. Carpentier and N. Darabiha. Proceedings of the Combustion Institute, 30:867-874, 2005.

[20] B. Fiorina, O. Gicquel, L. Vervisch, S. Carpentier and N. Darabiha. Combust. Flame, 140: 147-160, 2005.

[21] P. Domingo, L. Vervisch and K. Bray. Combust. Theory and Modeling, 6 529-551, 2002.

[22] N. Darabiha. Combust. Sci. Tech., 86:163-181, 1992.

[23] J. Luche. Elaboration of reduced kinetic models of combustion. Application to a kerosene mechanism., Ph.D. thesis, LCSR Orléans, 2003. 
Table 1: Different studied cases.

\begin{tabular}{ccccc}
\hline $\begin{array}{c}\text { Name } \\
\text { case }\end{array}$ & $\begin{array}{c}D_{l}^{f} \\
{[\mu \mathrm{m}]}\end{array}$ & $\begin{array}{c}\alpha_{l}^{f} \\
{[-]}\end{array}$ & $\begin{array}{c}n_{l}^{f} \\
{\left[1 / \mathrm{m}^{3}\right]}\end{array}$ & $\begin{array}{c}v_{g}^{f} \\
{[\mathrm{~m} / \mathrm{s}]}\end{array}$ \\
\hline REF. & 40 & $3.010^{-3}$ & $1.010^{+10}$ & 0.20 \\
\hline DIAMa & $\mathbf{7 0}$ & $3.010^{-3}$ & $\mathbf{1 . 8 7} \mathbf{1 0}^{+\mathbf{9}}$ & 0.20 \\
DIAMb & $\mathbf{1 4 0}$ & $3.010^{-3}$ & $\mathbf{0 . 2 3} \mathbf{1 0}^{+\mathbf{9}}$ & 0.20 \\
ALPHAa & 40 & $\mathbf{1 . 4} \mathbf{1 0}^{-\mathbf{3}}$ & $\mathbf{4 . 8 1} \mathbf{1 0}^{+\mathbf{9}}$ & 0.20 \\
ALPHAb & 40 & $\mathbf{0 . 6 8} \mathbf{1 0}^{-\mathbf{3}}$ & $\mathbf{2 . 3 3} \mathbf{1 0}^{+\mathbf{9}}$ & 0.20 \\
VELa & 40 & $3.010^{-3}$ & $1.010^{+10}$ & $\mathbf{0 . 5 0}$ \\
VELb & 40 & $3.010^{-3}$ & $1.010^{+10}$ & $\mathbf{1 . 0 0}$ \\
\hline
\end{tabular}

Table 2: Liquid kerosene properties.

\begin{tabular}{|c|c|}
\hline Liquid specific mass $\rho_{q}$ & $781 \mathrm{~kg} / \mathrm{m}^{3}$ \\
Latent heat of evaporation $L$ & $289.9 \mathrm{~kJ} / \mathrm{kg}$ \\
Boiling temperature & $478.9 \mathrm{~K}$ \\
Const. pressure heat capacity $c_{p, l}$ & $2.003 \mathrm{~kJ} /(\mathrm{kg} \mathrm{K})$ \\
\hline
\end{tabular}






Figure 1: The evaporation source term $n_{l} \dot{m}_{l}$ (continuous line) and the gaseous temperature (dashed line) as a function of the axial position in the reference spray flame.

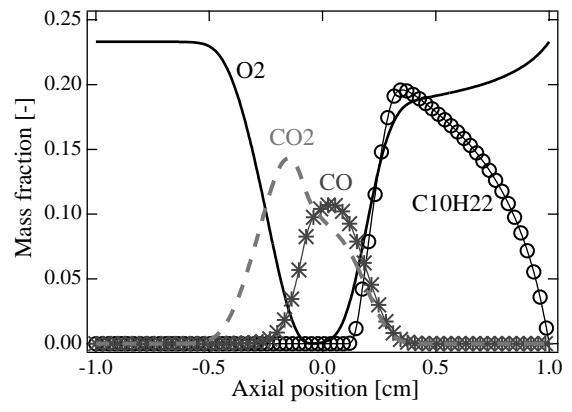

Figure 2: Species mass fraction profiles as a function of the axial position in the reference spray flame. 

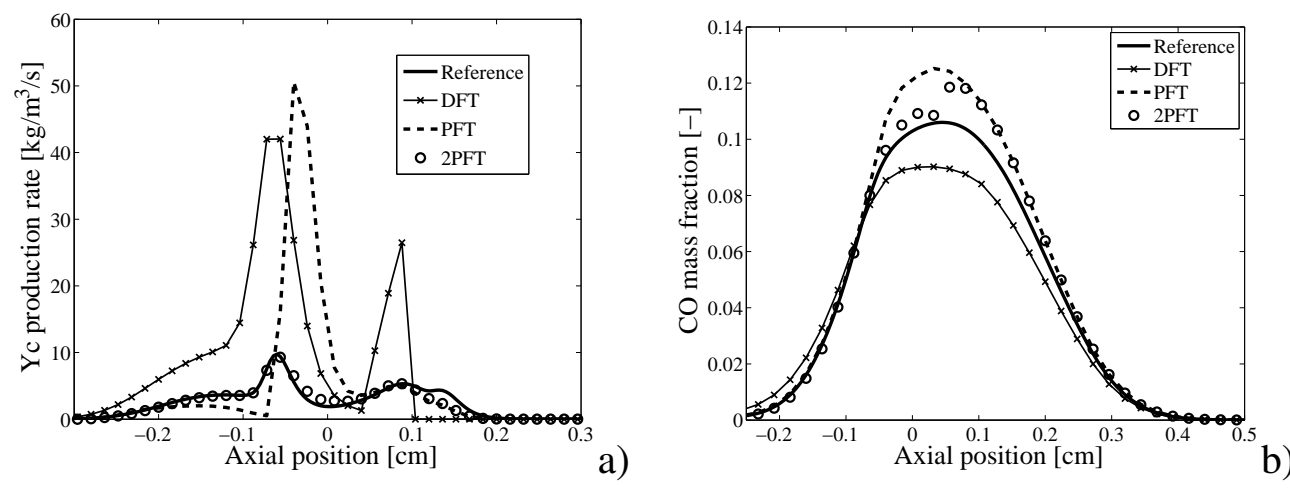

Figure 3: a) The production rate of the progress variable $\dot{\omega}_{Y_{c}}$ and b) the $C O$ mass fraction of the reference spray flame obtained with the detailed chemistry (black continous line) is compared to the a priori profiles for the three tabulated mechanisms: PFT premixed method (dashed gray line), DFT diffusion method (gray crosses), 2PFT technique (black circles).
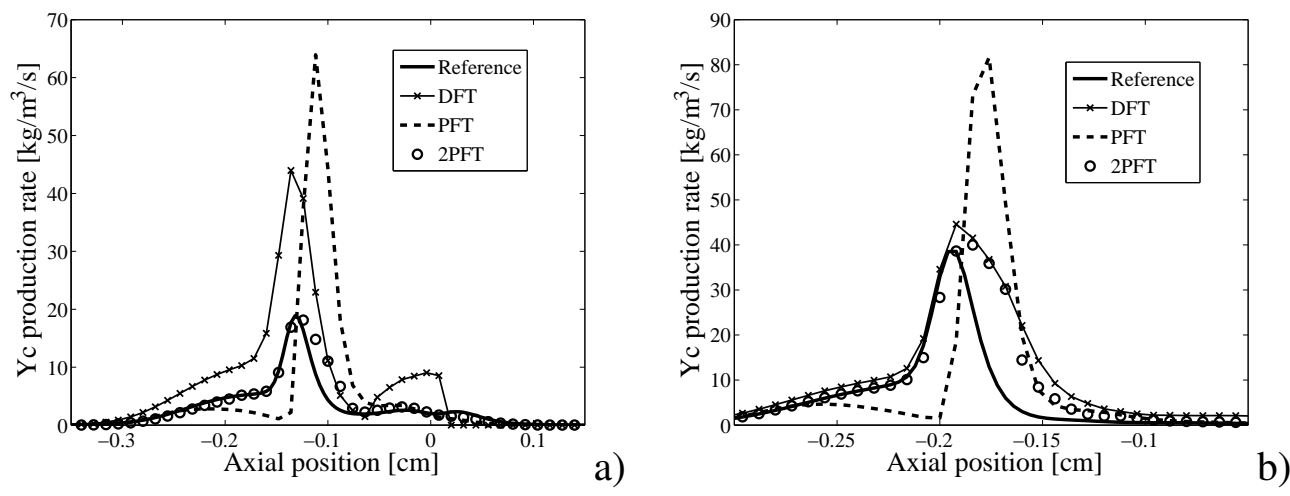

Figure 4: Production rates of the progress variable $\dot{\omega}_{Y_{c}}$ for a) $D_{l}^{f}=70 \mu \mathrm{m}$ and b) $D_{l}^{f}=140 \mu \mathrm{m}$. Lines and symbols are the same as in Fig. 3. 

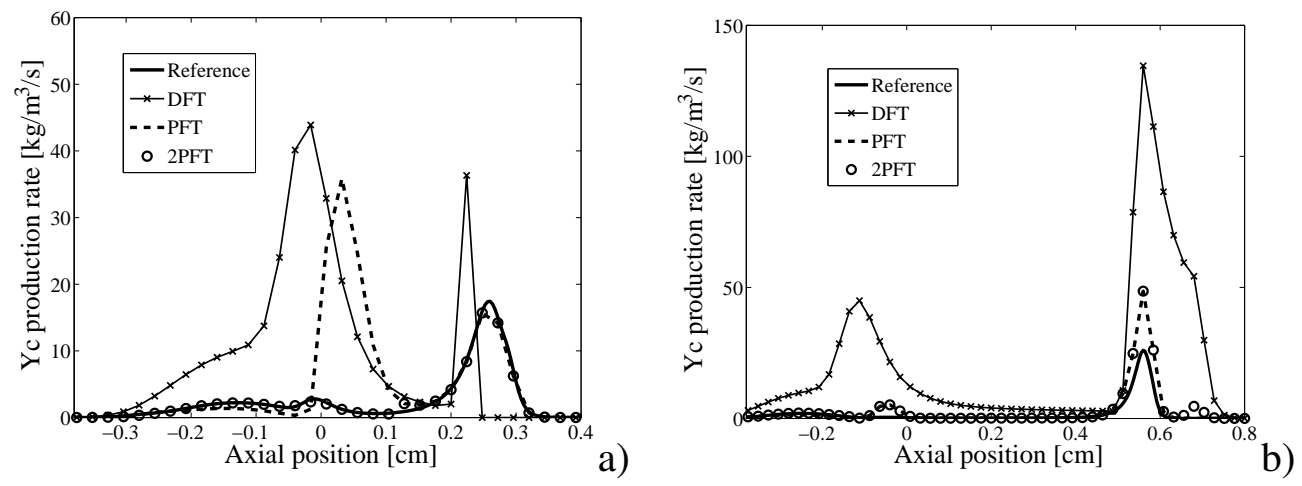

Figure 5: Production rates of the progress variable $\dot{\omega}_{Y_{c}}$ for injection liquid volume fraction equal to a) $\alpha_{l}^{f}=1.410^{-3}$ and b) $\alpha_{l}^{f}=0.6810^{-3}$ (continuous black line). Lines and symbols are the same as in Fig. 3.
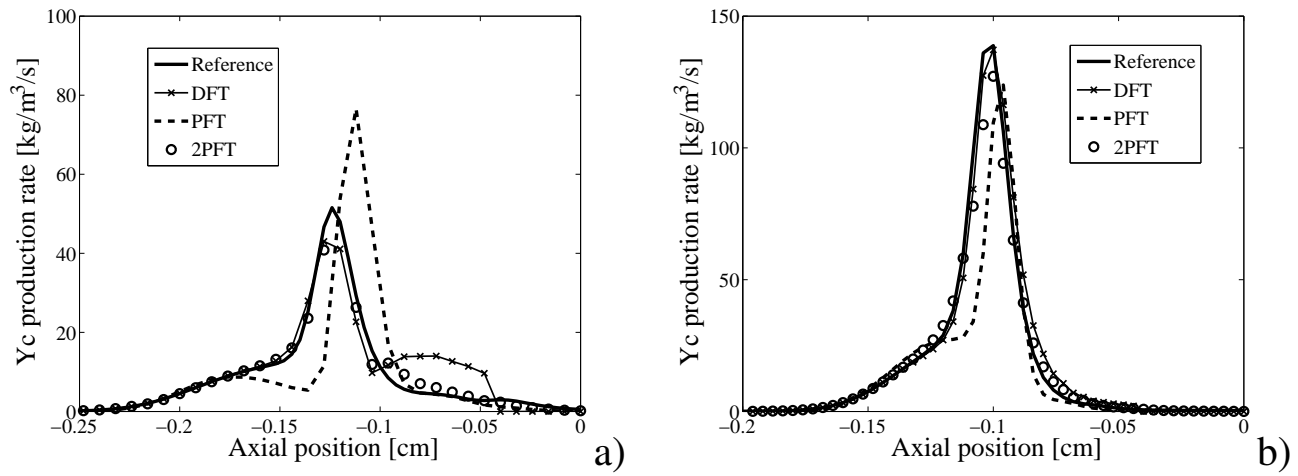

Figure 6: Production rates of the progress variable $\dot{\omega}_{Y_{c}}$ for injection velocity a) $v_{g}^{f}=v_{l}^{f}=0.5 \mathrm{~m} / \mathrm{s}$ and b) $v_{g}^{f}=v_{l}^{f}=1.0 \mathrm{~m} / \mathrm{s}$ (continuous black line). Lines and symbols are the same as in Fig. 3. 


\section{List of Figures}

1 The evaporation source term $n_{l} \dot{m}_{l}$ (continuous line) and the gaseous temperature (dashed line) as a function of the axial position in the reference

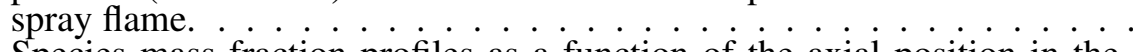

2 Species mass fraction profiles as a function of the axial position in the reference spray flame. . . . . . . . . . . . .

a) The production rate of the progress variable $\dot{\omega}_{Y_{c}}$ and b) the $C O$ mass fraction of the reference spray flame obtained with the detailed chemistry (black continous line) is compared to the a priori profiles for the three tabulated mechanisms: PFT premixed method (dashed gray line), DFT diffusion method (gray crosses), 2PFT technique (black circles). . . . . . 20

4 Production rates of the progress variable $\dot{\omega}_{Y_{c}}$ for a) $D_{l}^{f}=70 \mu \mathrm{m}$ and b) $D_{l}^{f}=140 \mu \mathrm{m}$. Lines and symbols are the same as in Fig. 3. . . . . . . 20

5 Production rates of the progress variable $\dot{\omega}_{Y_{c}}$ for injection liquid volume fraction equal to a) $\alpha_{l}^{f}=1.410^{-3}$ and b) $\alpha_{l}^{f}=0.6810^{-3}$ (continuous black line). Lines and symbols are the same as in Fig. 3. . . . . . . . .

6 Production rates of the progress variable $\dot{\omega}_{Y_{c}}$ for injection velocity a) $v_{g}^{f}=$ $v_{l}^{f}=0.5 \mathrm{~m} / \mathrm{s}$ and b) $v_{g}^{f}=v_{l}^{f}=1.0 \mathrm{~m} / \mathrm{s}$ (continuous black line). Lines and symbols are the same as in Fig. 3. . . . . . . . . . . . . . 21 